\title{
Análise Atmosférica dos Eventos de Efeito Secundário do Buraco de Ozônio Antártico Sobre o Sul do Brasil em 2012. Parte 1: Identificação dos Eventos e Análise da Dinâmica da estratosfera
}

\section{Atmospheric Analysis of the Events Secondary Effect of Antarctic Ozone Hole Over the South of} Brazil in 2012. Part 1: Identification of the Events and Analysis of Dynamic in the stratosphere

Lucas Vaz Peres ${ }^{1}$, Nicolle Cordero Simões dos Reis²; Letícia de Oliveira dos Santos' ${ }^{2}$, Gabriela Dornelles Bittencourt ${ }^{2}$, André Passaglia Schuch ${ }^{3}$, Vagner Anabor ${ }^{1,2}$, Damaris Kirsch Pinheiro ${ }^{1,4}$, Nelson Jorge $\mathrm{Schuch}^{5}$ e Neusa Maria Paes Leme ${ }^{6}$

\author{
${ }^{1}$ Programa de Pós Graduação em Meteorologia, UFSM, Santa Maria, RS, Brasil; \\ lucasvazperes@gmail.com,vanabor@gmail.com; \\ ${ }^{2}$ Graduação em Meteorologia, UFSM, Santa Maria, RS, Brasil; \\ nicolle_csr@hotmail.com; deoliveiraaicitel@gmail.com; gabezinha08@hotmail.com \\ ${ }^{3}$ Programa de Pós Graduação em Biodiversidade Animal, UFSM Santa Maria, RS, Brasil; \\ schuchap@gmail.com \\ ${ }^{4}$ Departamento de Engenharia Química, UFSM, Santa Maria, RS, Brasil; \\ damariskp@gmail.com \\ ${ }^{5}$ Centro Regional Sul de Pesquisas Espaciais - CRS/CCR/INPE-MCTI, Santa Maria, RS, Brasil; \\ njschuch@lacesm.ufsm.br \\ ${ }^{6}$ INPE - Centro Regional do Nordeste - CRN/ INPE-MCTI, Natal, RN, Brasil. \\ neusa_paesleme@yahoo.com.br
}

\begin{abstract}
Resumo
Na presente estudo foram identificados os eventos de Efeito Secundário do Buraco de Ozônio Antártico ocorridos sobre o Sul do Brasil no ano de 2012. Para isso, foram analisados os dados médios diários da coluna total de ozônio obtidos através de Espectrofotômetro Brewer, instalado no Observatório Espacial do Sul - OES/CRS/INPE - MCTI (29,4 ${ }^{\circ}$; ; 53,8 $\left.{ }^{\circ} \mathrm{O} ; 488,7 \mathrm{~m}\right)$ e pelos instrumentos de satélite Total Ozone Mapping Spectrometer (TOMS) e Ozone Monitoring Instrument (OMI), buscando dias de quedas no conteúdo de ozônio. Para estes dias, foram analisados mapas de vorticidade potencial confeccionados utilizando dados de reanálise fornecidos pelo NCEP/NCAR e trajetórias retroativas confeccionadas através do modelo Hysplit da NOAA, a fim de verificar a origem polar da massa de ar pobre em ozônio. Além disso, foi também realizada uma análise complementar através das imagens do conteúdo de ozônio do satélite OMI, verificando-se a atuação do Buraco de Ozônio na região Antártica e sua conexão com o Sul do Brasil. A metodologia empregada mostrou-se eficaz na identificação de 2 eventos de Efeito Secundário do Buraco de Ozônio Antártico sobre o Sul do Brasil, os quais apresentaram uma queda média de 12,1 2,3\% no conteúdo de ozônio.
\end{abstract}

Palavras-chave: Efeito Secundário do Buraco de Ozônio Antártico. Espectrofotometro Brewer.

\begin{abstract}
In this study were identified events of Influence of the Antarctic ozone hole over southern Brazil in the year 2012. For this, we analyzed the daily average data of total ozone column obtained through Brewer Spectrophotometer, installed in the southern Space Observatory-OESCRSINPEMCTI (29.4 S; 53.8; $488 \mathrm{~m}$ ) and by satellite instruments Total Ozone Mapping Spectrometer (TOMS) and Ozone Monitoring Instrument $(O M I)$, seeking days of falls in the ozone content. For these days, we analyzed potential vorticity maps made using Reanalysis data provided by NCEPNCAR and retroactive trajectories made through the Hysplit model of NOAA, in order to verify the origin of the polar air mass poor in ozone. In addition, it was also conducted a complementary analysis through the images of the ozone content of the OMI satellite, verifying the performance of the Antarctic Ozone Hole in polar regions and its connection with the Southern Brazil. The methodology used was shown to be effective in the identification of 2 events of side effect of the Antarctic ozone hole over the South of Brazil, which showed a fall of $12.1 \pm 2.3$ average in the ozone content.
\end{abstract}




\section{Introdução}

O transporte meridional do ozônio estratosférico é um dos fatores essenciais para a concentração deste constituinte atmosférico em uma determinada região do planeta (Gettelman et al., 2011; Ploeger et al., 2012; Albers e Nathan, 2012), sendo muito estudo a partir da utilização da variável Vorticidade Potencial, que se correlacionam com o transporte de constituintes químicos traços como o ozônio, óxido nitroso e vapor d água (Schoeberl, 1989) sobre superfícies isentrópicas na baixa estratosfera, estudos estes apontado primeiramente por Danielsen em 1968 (Lary et al., 1995).

Esta variável tem um importante papel traçador na dinâmica de massas de ar de grande escala, comportando-se como uma superfície material onde a temperatura potencial é conservada (Hoskins et al., 1985).

Sua utilização foi largamente difundida em estudos de trocas entre a estratosfera e a troposfera em região onde há intrusão de ar estratosférico para dentro da troposfera e vice versa (Holton et al.,1995; Sthol et al., 2003; Jing et al., 2005; Sprenger et al., 2007 e Clain et al., 2010), sendo utilizada também em estudos de transporte horizontal de massas de ar e seu conteúdo de ozônio na estratosfera (Semane et al., 2006 e Bencherife et al. 2011).

A massiva destruição do conteúdo de ozônio durante as primaveras do Hemisfério Sul, denominada de "Buraco de Ozônio Antártico" (Chubachi, 1984; Farman et al., 1985 e Solomon, 1999) causa influência direta sobre o conteúdo de ozônio sobre regiões polares e suas proximidades, devido a passagem de sua borda sobre estas regiões, causando drásticas reduções do conteúdo de ozônio e aumento dos níveis de radiação ultravioleta que chega a superfície (Larry et al. 1995; Kirchoff et al. 1997; Perez et al. 1998 e Casiccia et al., 2008).

Entretanto, seus efeitos podem afetar regiões de médias e baixas latitudes, causando diminuições temporárias na coluna total de ozônio sobre estas regiões (Prather e Jaffe,1990; Waugh, 1993; Waugh et al., 1994; Manney et al., 1994).

Massas de ar pobres em ozônio, podem se desprender do interior do vórtice polar antártico, borda do Buraco de Ozônio e serem transportadas pelos filamentos polares sobre estas regiões (Nash et al., 1996, ; Marchand et al. 2005), em um fenômeno denominado de "Efeito secundário do Buraco de Ozônio Antártico" que ocasionando uma queda temporária do conteúdo de ozônio, observado primeiramente por Kirchhoff et al. (1996) sobre o Sul do Brasil, e sendo frequentemente observado sobre a América do Sul (Perez e Jaque, 1998; Perez et al., 2000, Paznino et al., 2008), Sul do Brasil (Pinheiro et al., 2011; 2012; Peres et al.,2011; 2012), Sul do continente africano (Semane et al., 2006; Sivakumar et al., 2007) e Nova Zelândia (Brinksma et al., 1998).

Estudos recentes apontaram que os cinco eventos de Efeito Secundário do Buraco de Ozônio Antártico ocorridos entre os anos de 2008 e 2009 causaram quedas temporárias médias na coluna total de ozônio de 9,7 $\pm 3,3 \%$ (Pinheiro et al., 2011), já os sete eventos identificados entre 2010 e 2011 causaram queda médias de $6.3 \pm$ 2.1\% (Pinheiro et al., 2012), ambas quedas em relação as medias mensais climatológicas do mês de ocorrência de cada evento.

Comprovando a importância deste tipo de estudo, reduções de $1 \%$ no conteúdo de ozônio podem causar aumento de até $2 \%$ na radiação ultravioleta incidente sobre a superfície terrestre. Já sobre o Sul do Brasil uma mesma queda de $1 \%$ no conteúdo de ozônio causa aumento médio de $1,2 \%$ na radiação ultravioleta incidente (Guarnieri et al., 2004).

O presente estudo tem por objetivo, identificar e analisar a dinâmica das massas de ar na estratosfera durante a ocorrência dos eventos de "Efeito Secundário do Buraco de Ozônio Antártico" sobre o Sul do Brasil, no ano de 2012, indicando, a origem destas massas de ar pobre em ozônio, e intensidade das quedas observadas.

\section{Metodologia}

A identificação dos dias de ocorrência de eventos de "Efeito Secundário do Buraco de Ozônio Antártico" sobre o Sul do Brasil, no ano de 2012 se deu através da análise de quatro principais ingredientes (Pinheiro et al., 2011; 2012; Peres 2013 ). 
Primeiramente, analisam-se os dados do monitoramento de longo prazo, 1979 a 2012, da média diária da coluna total de ozônio sobre o Sul do Brasil, obtido pelos Espectrofotômetros Brewer modelo MKIV \#081 durante o período de 1992 - 2000, modelo MKII \#056 de 2000 - 2002 e modelo MKIII \#167 de 2002 até o momento, instalados no Observatório Espacial do Sul OES/CRS/INPE - MCTI (29, $\left.4^{\circ} \mathrm{S} ; 53,8^{\circ} \mathrm{O} ; 488,7 \mathrm{~m}\right)$, em São Martinho da Serra, a partir de um convênio entre a Universidade Federal de Santa Maria (UFSM) e o Instituto Nacional de Pesquisas Espaciais (INPE/MCTI) e também, disponível a partir de 1979, pelos instrumentos de satélite Total Ozone Mapping Spectrometer (TOMS), que esteve a bordo dos satélites Nimbus-7, Meteor-3 e Earth Probe da National Aeronautics and Space Agency (NASA); e Ozone Monitoring Instrument (OMI), o qual substituiu os dados do TOMS desde 2006, ambos dados de satélites coletados para a mesma localização do Observatório Espacial do Sul.

A complementação de dados de satélite a séries de dados do equipamento de solo Espectrofotometro Brewer é possível devido à diferença de medida entre os dois tipos de equipamentos serem menor que $3 \%$, possibilitando a formação da série de dados entre 1979 e 2012 (Peres, 2013).

Esta análise consiste da verificação dos dias no ano de 2012, em que houve queda temporária extrema no conteúdo de ozônio abaixo do limite estipulado de média climatológica do mês menos 1,5 do seu respectivo desvio padrão $(\mu \mathrm{i}-1,5 \sigma \mathrm{i})$ entre os meses de agosto e novembro (Pinheiro et al., 2011 e 2012), período de atuação do Buraco de Ozônio Antártico (Solomon, 1999).

Para os dias de queda na coluna total de ozônio, foram gerados mapas de Vorticidade Potencial e vento para a superfície isentrópica de $620 \mathrm{~K}$ de temperatura potencial, representando as alturas de $24 \mathrm{~km}$, nível preferencial de chegada deste tipo de massas de ar estratosférica (Peres, 2013), a partir de parâmetros diários das componentes meridional (v-wind) e zonal (uwind) do vento e de temperatura para os níveis de pressão 1000, 925, 850, 700, 600, 500, 400, 300, 250, 200, 150, 100, 70, 50, 30, 20, $10 \mathrm{hPa}$, fornecidos pelo National Centers for Environmental Prediction/National Atmospheric Research
(NCEP/NCAR), dados de reanálise disponíveis em

http://www.cdc.noaa.gov/cdc/reanalysis/reanaly sis.shtml (Kalnay et al., 1996) e gerados no software de domínio público GrADS (Grid Analysis and Display System).

Para uma determinada região, massas de ar estratosféricas possuem origem polar se é verificado um aumento de Vorticidade Potencial Absoluta dos dias anteriores para o dia em questão, e uma diminuição se a origem for equatorial (Semane et al., 2006), sendo este tipo de análise utilizada na descrição da dinâmica da estratosfera durante a ocorrência deste tipo de fenômeno (Pinheiro et al., 2011,2012).

Trajetórias retroativas das massas de ar foram confeccionadas através do modelo HYSPLIT (HYbrid Single-Particle Lagrangian Integrated Trajectory) da National Oceanic and Atmospheric Administration (NOAA), com a finalidade de confirmação da origem das massas pobre em ozônio.

Este modelo pode ser rodado interativamente na web e também utilizado os dados de reanálise do NCEP/NCAR (Gupta et. al., 2007), sendo disponível no seguinte link: http://www.arl.noaa.gov/ready/open/traj.html, servindo como confirmação para a análise isentrópica, complementando a visualização da origem das massas de ar realizada para seis dias anteriores à ocorrência do evento para as alturas de 20,24 e $28 \mathrm{~km}$ de altura, verificando se ela passou pela região polar.

Complementando a análise, é realizada a observação das imagens do conteúdo de ozônio do instrumento de satélite OMI da NASA, disponível em http://ozoneaq.gsfc.nasa.gov/, para os referidos dias de queda na coluna de ozônio sobre o Sul do Brasil, verificando a existência de conexão entre esta região e o Buraco de Ozônio Antártico, através da ejeção de massas de ar pobre em ozônio para fora do vortice polar Antártico (Pinheiro et al., 2011; 2012; Peres et al.,2011, 2012).

\section{Resultados e Discussões}

Foram calculados os valores das médias climatológicas, desvio padrão e o valor limite de 
média climatológica menos 1,5 desvios padrão ( $\mu \mathrm{i}-1,5 \sigma \mathrm{i})$, utilizados para selecionar os eventos de queda na coluna de ozônio, para os meses de agosto, setembro, outubro e novembro no período entre 1979 e 2012 sobre a região do Observatório Espacial do Sul, sendo este valores considerados normais para regiões de médias latitudes como o Sul do Brasil (London, 1985) e apresentados na tabela 1 .

Tabela 4.3: Médias climatológicas da coluna total de ozônio e seus respectivos desvios padrão e limite de média menos 1,5 desvios padrão ( $\mu$ i$1,5 \sigma i)$ para os meses de agosto, setembro, outubro e novembro, no período entre 1979 e 2011, para a região do Observatório Espacial do $\operatorname{Sul}\left(29,4^{\circ} \mathrm{S} ; 53,8^{\circ} \mathrm{O} ; 488,7 \mathrm{~m}\right)$.

\begin{tabular}{cccc}
\hline Mês & O3 & SD & $\boldsymbol{\mu} \mathbf{i}-\mathbf{1 , 5 \sigma i}$ \\
& (UD) & (UD) & (UD) \\
\hline Agosto & 292,38 & 11,65 & 274,91 \\
Setembro & 298,87 & 9,80 & 284,17 \\
Outubro & 292,73 & 9,85 & 277,95 \\
Novembro & 287,47 & 9,89 & 272,63 \\
\hline
\end{tabular}

Durante o ano de 2012 foram identificados dois eventos de "Efeito Secundário do Buraco de Ozônio Antártico" sobre o Sul do Brasil que atenderam aos quatro ingredientes conforme descrito na metodologia, totalizando uma queda média de 12,1 $\pm 2,3 \%$ no conteúdo de ozônio. Os eventos serão apresentados detalhadamente a seguir:

\subsection{Evento de 14 de setembro de 2012}

Analisando os dados da coluna total de ozônio, para o período entre os dias 10 e 18 de setembro de 2012, Figura 1, observa-se uma queda temporária no conteúdo de ozônio abaixo do limite de média mensal climatológica menos 1,5 desvio padrão, onde o valor mais baixo no conteúdo de ozônio foi alcançado no dia 14 de setembro, de 267,8 UD, representando uma redução de 10,4\% em relação à média climatológica do mês de setembro, 298,58 \pm 9,8 DU, aumentando seus valores nos dias seguintes.

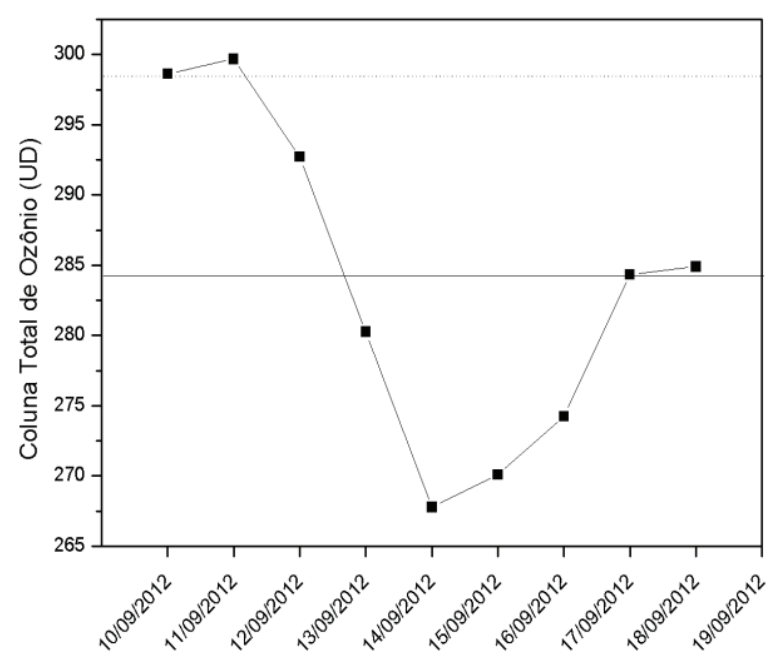

Figura 1 - Valores da coluna total de ozônio entre os dias 10 e 18 de setembro de 2012 para a região do Observatório Espacial do Sul. Linha tracejada representa o valor da média climatológica e a linha contínua representa valor limite de média climatológica menos 1,5 desvios padrão para o mês de setembro

A dinâmica da estratosfera é analisada a partir dos mapas de vorticidade potencial no nível isentrópico de $620 \mathrm{~K}$ de temperatura potencial, onde observa-se (Figura 2), que no dia 13 de setembro de 2012 (a), a massa de ar com vorticidade potencial absoluta maior já se encontrava sobre o centro-norte da Argentina e Uruguai. Os ventos predominantemente de Sul, envoltos em uma circulação ciclônica, proporcionaram o avanço desta massa de ar que se instalou definitivamente sobre o Sul do Brasil no dia 14 de setembro de 2012 (b) verificado pelo aumento de vorticidade potencial absoluta.

A trajetória retroativa da massa de ar, confeccionada pelo modelo Hysplit da NOAA, do dia 14 de setembro (c), e a imagem do conteúdo de ozônio do satélite OMI, do dia 13 de setembro (d), complementam a análise, evidenciando a origem polar da massa de ar e a conexão entre o Buraco de Ozônio com o Sul do Brasil durante o evento que ocasionou a queda temporária na coluna de ozônio, confirmando a ocorrência do evento de Efeito Secundário do Buraco de Ozônio Antártico sobre o Sul do Brasil do dia 14 de setembro de 2012. 
Potencial Vorticity, 13/SEP/2012, 620k

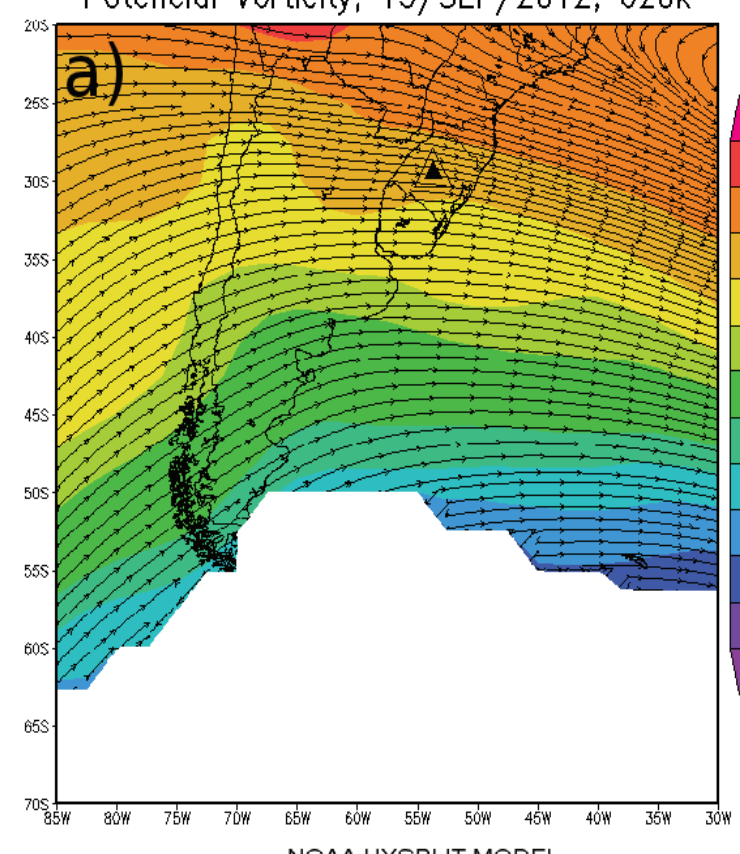

GDAS Metcorological Data

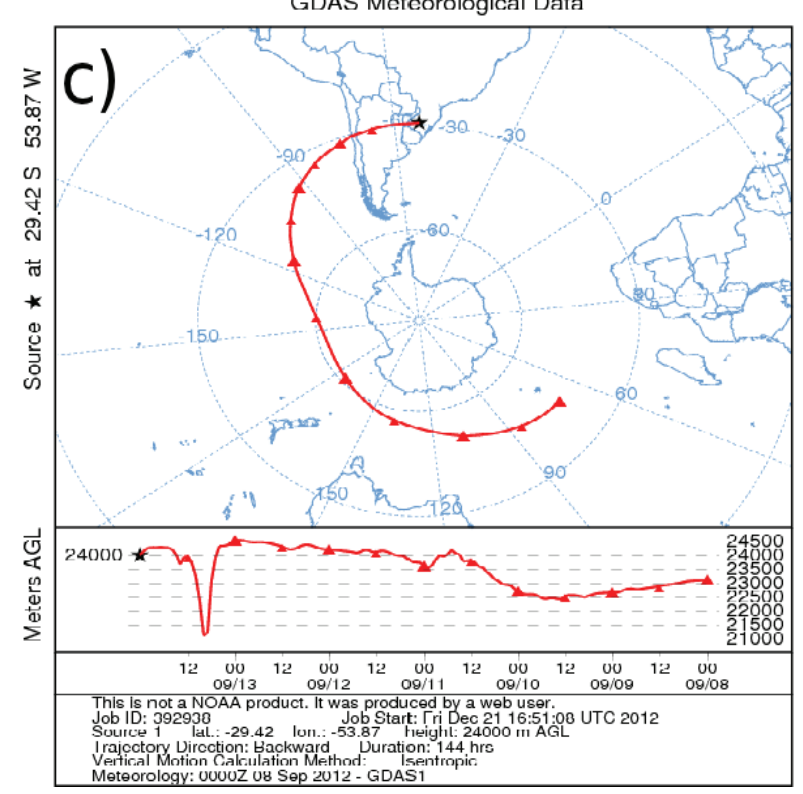

Potencial Vorticity, 14/SEP/2012, 620k

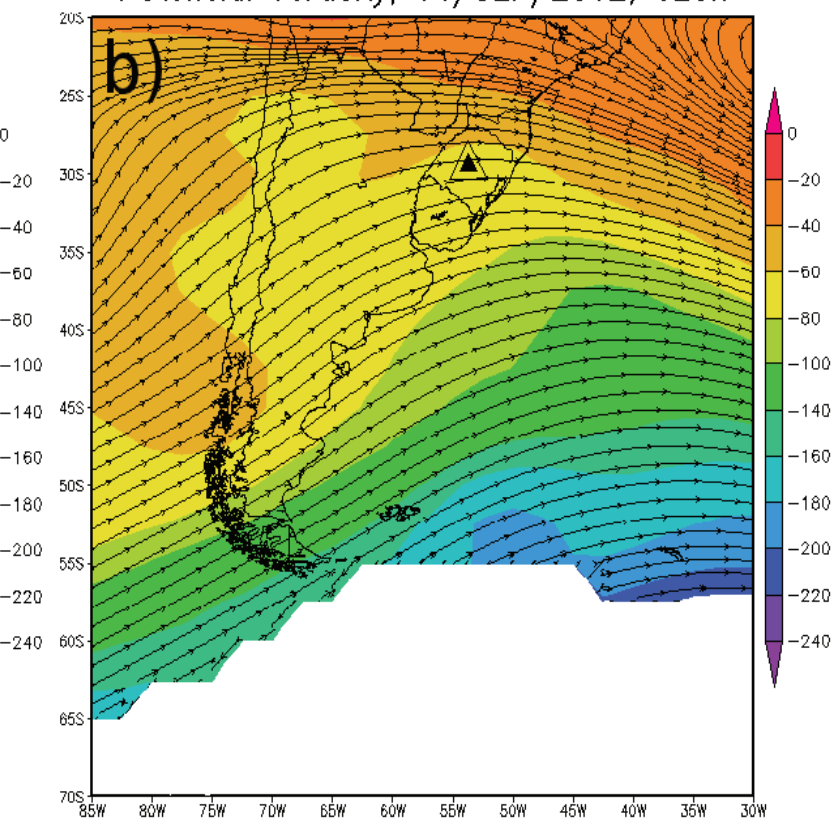

OMll Total Ozone Sep 13, 2012
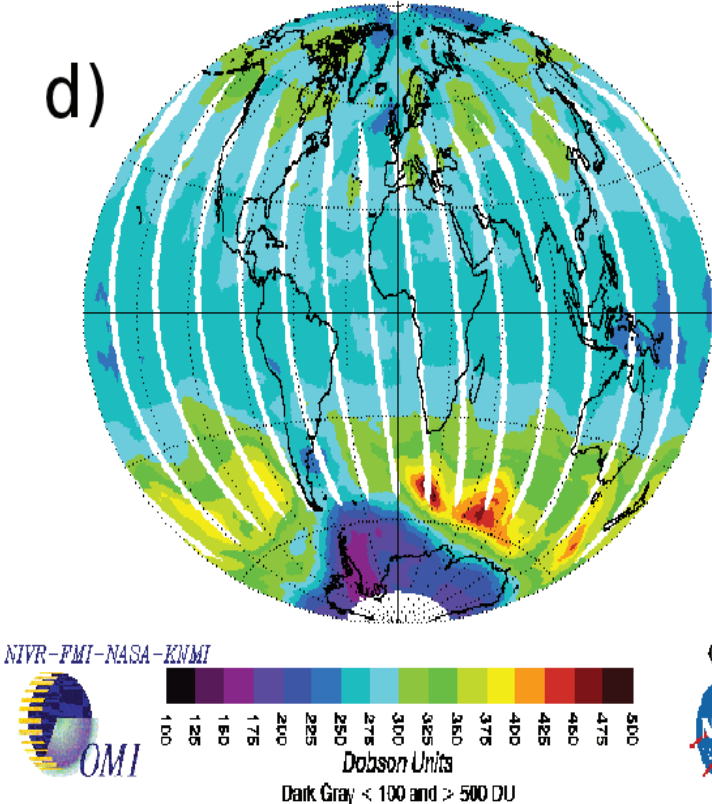

GSFC

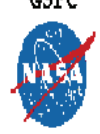

Figura 2 - Vorticidade Potencial e vento para 13 (a) e 14 (b) de setembro de 2012 a 620K. Trajetória retroativa da massa de ar (c) e imagem do satélite OMI (d) para os dias 14 e 13 de setembro de 2012, respectivamente

\subsection{Evento de 14 de Outubro de 2012}

Os dados da coluna total de ozônio para o período entre os dias 11 e 18 de outubro de 2012 foram analisados, Figura 3, observando-se uma queda temporária no conteúdo de ozônio abaixo do limite de média mensal climatológica, do período entre 1979 e 2011, menos 1,5 desvio padrão, que alcançou, no dia 14 de setembro, dia da menor queda, o valor de 252,6 UD, representando uma redução de 13,7\% em relação à média climatológica do mês de outubro, 292,7 \pm 9,85 UD, aumentando seus valores nos dias seguintes.

Analisando a dinâmica da estratosfera através dos mapas de vorticidade potencial no nível isentrópico de $620 \mathrm{~K}$ de temperatura potencial, Figura 4, observa-se que, no dia 13 de outubro de 2012 (a), uma intensa massa de ar com vorticidade potencial absoluta maior encontrava-se sobre o centro-norte da Argentina e Uruguai e os ventos predominantemente de 
Sul, envoltos em uma circulação ciclônica, proporcionaram o avanço desta massa de ar sobre o Sul do Brasil no dia 14 de outubro de 2012 (b).

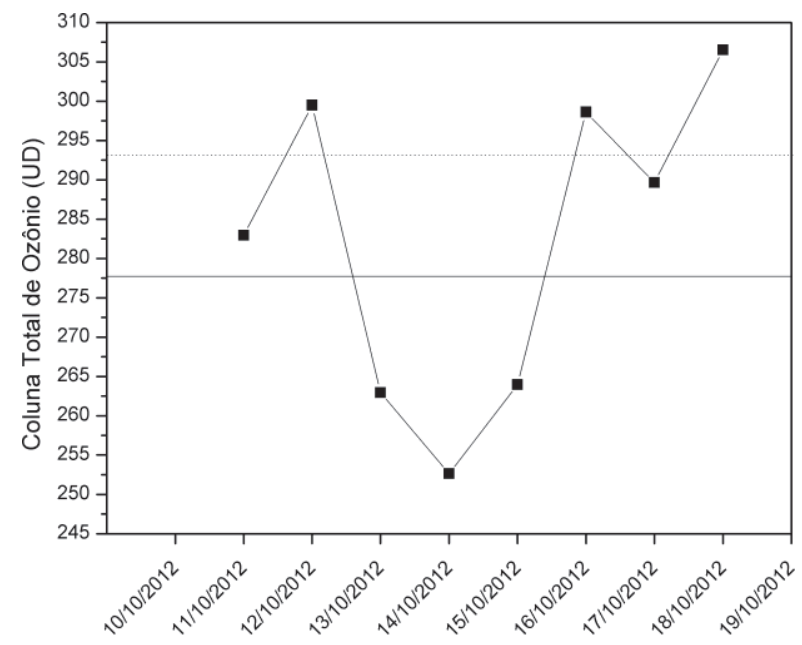

Figura 3 - Valores da coluna total de ozônio entre os dias 11 e 18 de outubro de 2012 para a região do Observatório Espacial do Sul. Linha tracejada representa o valor da média climatológica e a linha contínua representa valor limite de média climatológica menos 1,5 desvios padrão para o mês de outubro.

A trajetória retroativa da massa de ar, confeccionada pelo modelo Hysplit da NOAA, do dia 14 de setembro (c), mostra sua passagem por cima do continente Antártico e a imagem do conteúdo de ozônio do satélite OMI, do dia 13 de setembro (d), complementa a análise, mostrando a conexão entre o Buraco de Ozônio com o Sul do Brasil, comprovando a origem polar da massa de ar pobre em ozônio que atingiu o Sul do Brasil e ocasionou a queda temporária na coluna de ozônio, confirmando a ocorrência do evento de Efeito Secundário do Buraco de Ozônio Antártico sobre o Sul do Brasil no dia 14 de outubro de 2012.

\section{Discussões}

Eventos semelhantes de desprendimento de massas de ar estratosférias pobres em ozônio oriundas do Buraco de Ozônio da Antártida e seu transporte em direção a médias latitude através de filamentos polares (Nash et al., 1996, ; Marchand et al. 2005), também foram observadas sobre a América do Sul (Perez e Jaque, 1998; Perez et al., 2000, Paznino et al., 2008), Sul do continente africano (Semane et al., 2006;
Sivakumar et al., 2007) e Nova Zelândia (Brinksma et al., 1998).

Sobre o Sul do Brasil este tipo de fenômeno vem sendo constantemente observado, causando queda temporária média na coluna total de ozônio de 9,7 $\pm 3,3 \%$ entre os cinco eventos identificados entre 2008 e 2009 (Pinheiro et al., 2011) e de $6.3 \pm 2.1 \%$ entre os sete eventos de 2010 e 2011 (Pinheiro et al., 2012), comprovando a importância do tema devido a reduções de $1 \%$ no conteúdo de ozônio causarem aumento médio de $1,2 \%$ na Radiação Ultravioleta sobre esta região (Guarnieri et al., 2004). Além disso, costumam estar associados a condições de tempo sinóticas na troposfera, principalmente relacionadas a condições pós frontais de massas de ar estáveis (Peres et al,. 2011, 2012).

Tanto as condições sinóticas troposféricas quanto a influência da diminuição do conteúdo de ozônio sobre a radiação ultravioleta para os eventos de Efeito Secundário do Buraco de Ozônio Antártico sobre o Sul do Brasil no ano de 2012 serão assunto nas próximas partes desta pesquisa respectivamente.

\section{Conclusões}

A partir da metodologia empregada de analise dos dados da coluna total de ozônio, dos mapas de Vorticidade Potencial e vento para a superfície isentrópica de $620 \mathrm{~K}$ de temperatura potencial, das trajetória retroativa do modelo Hysplit da NOAA e as imagem do conteúdo de ozônio do instrumento de satélite OMI, identificou-se a ocorrência de dois eventos Efeito Secundário do Buraco de Ozônio Antártico sobre o Sul do Brasil no ano de 2012, um no dia 14 de setembro e um no dia 14 de outubro, totalizando uma queda média de 12,1 $\pm 2,3 \%$ no conteúdo de ozônio.

O evento do dia 14 de setembro de 2012 teve uma queda de $10,4 \%$ em relação à média climatológica do mês de setembro, enquanto que no dia 14 de outubro de 2012 a queda foi de $13,7 \%$ em relação à média climatológica do mês de outubro.

Em ambos os casos, as quedas na coluna de ozônio mencionadas acima foram ocasionadas por massa de ar pobre em ozônio 
originárias da região do Buraco de Ozônio Antártico que atingiram o Sul do Brasil, observadas pela dinâmica da estratosfera devido ao aumento de vorticidade potencial absoluta, pela passagem dessas massas de ar pelo interior do circulo polar Antártico observado nas

Potencial Vorticity, $13 / 0 \mathrm{CT} / 2012,620 \mathrm{k}$

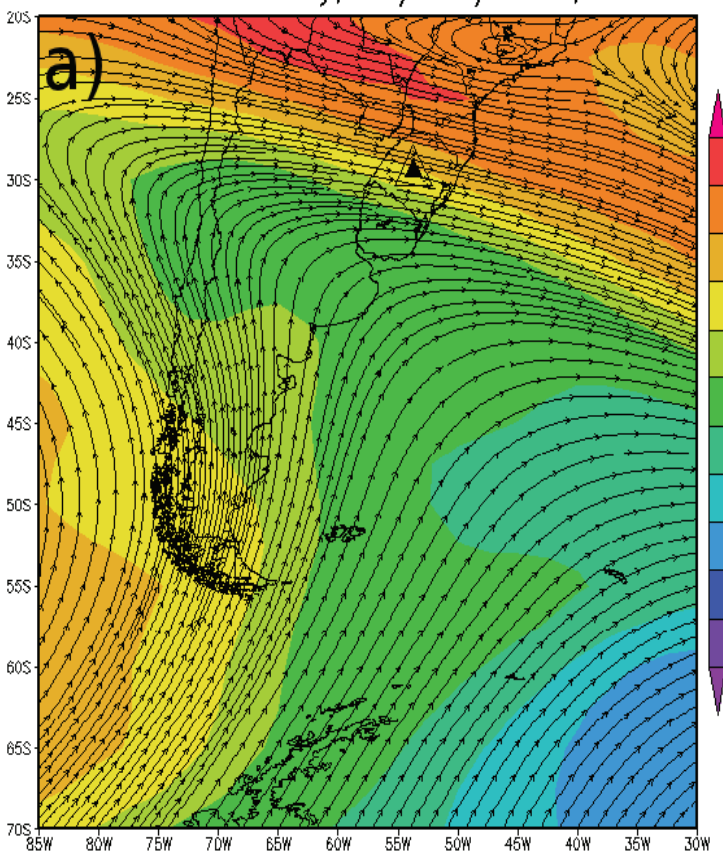

NOAA HYSPLIT MODEL

Backward trajectory ending at 0000 UTC 14 Oct 12 GDAS Meteorological Data

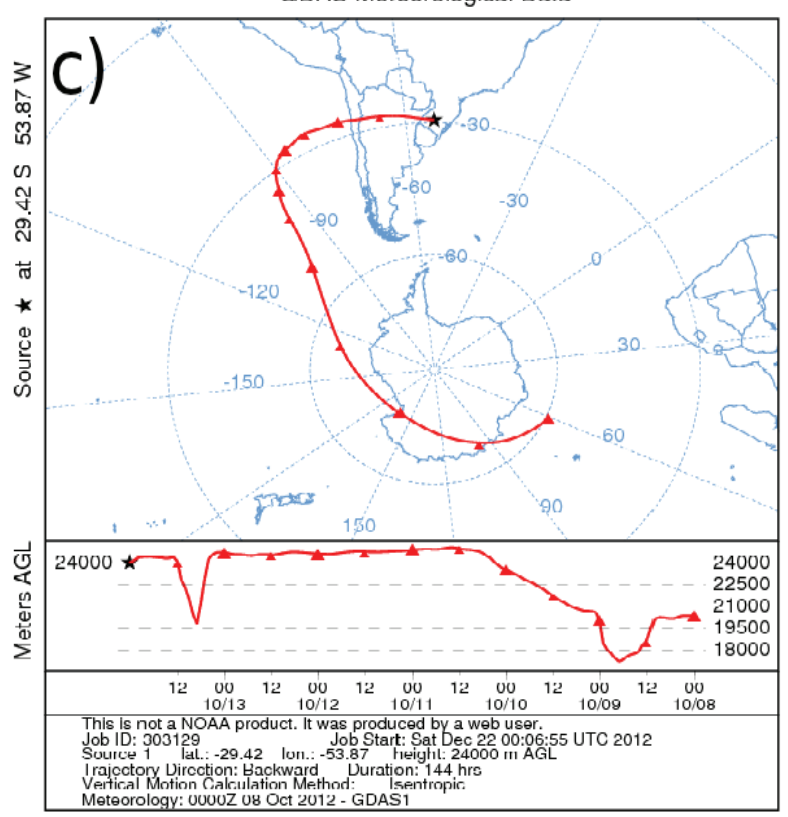

trajetórias retroativas do modelo Hysplit e pela imagem do conteúdo de ozônio do instrumento de satélite OMI, onde mostra a existência de uma conexão entre o Buraco de Ozônio Antártico e o Sul do Brasil.

Potencial Vorticity, 14/0CT/2012, 620k

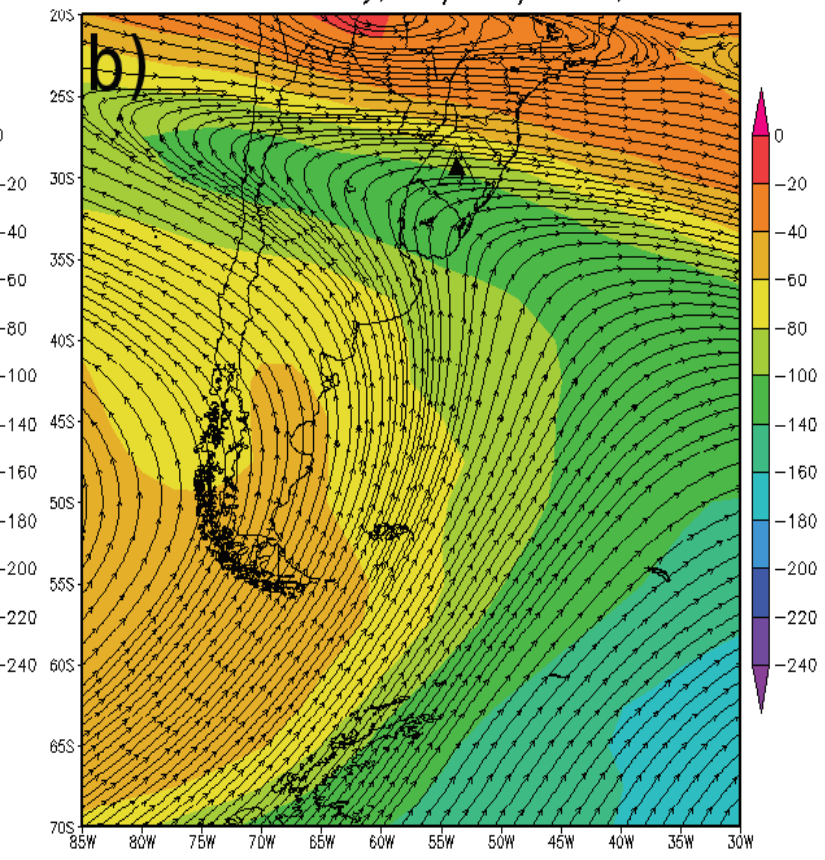

OMl Total Ozone Oct 13, 2012
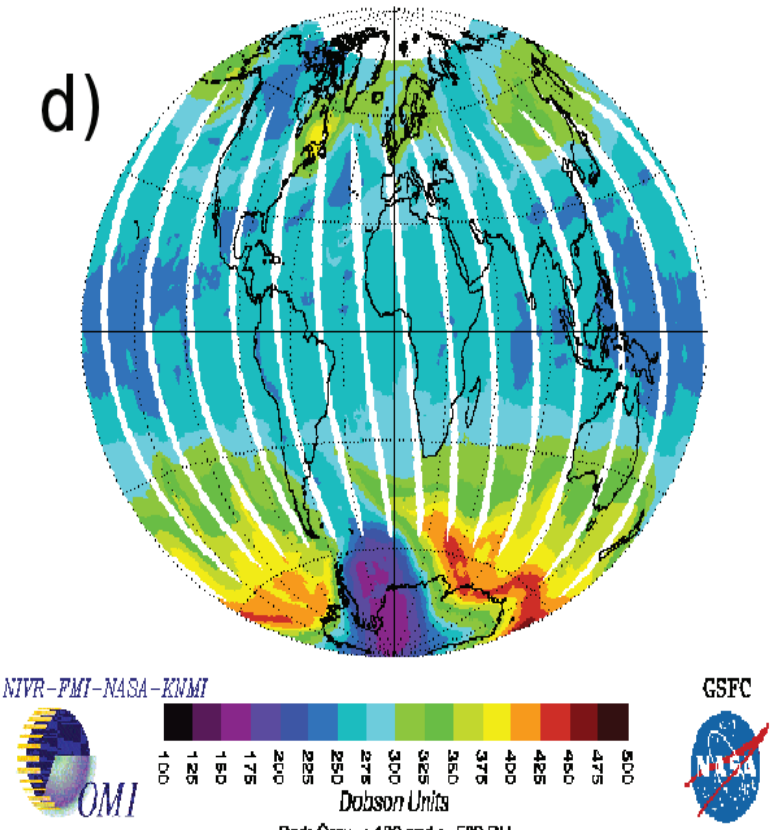

Figura 4 - Vorticidade Potencial e vento para 13(a) e 14 (b) de outubro de 2012 a 620K e imagem do satélite OMI (c) para 13 de outubro de 2012. 


\section{Agradecimentos}

Este trabalho integra o Programa de PósGraduação em Meteorologia da Universidade Federal de Santa Maria (UFSM) juntamente com o Instituto Nacional de Ciência e Tecnologia Antártico de Pesquisas Ambientais (INCT-APA), que recebe apoio científico e financeiro do Conselho Nacional de Pesquisa e Desenvolvimento (CNPq process: $n^{\circ}$ 574018/2008-5) e Fundação Carlos Chagas de Amparo à Pesquisa do Estado do Rio de Janeiro (FAPERJ $\mathrm{n}^{\circ}$ E-16/170.023/2008). Os autores também agradecem o apoio dos Ministérios da Ciência, Tecnologia e Inovação (MCTI), do Ministério do Meio Ambiente (MMA) e da Comissão Interministerial da para os Recursos do Mar (CIRM). Agradecimentos também para FAPEREGS / CAPES pela Bolsa de fomento, a NASA / TOMS e NCEP / NCAR pelos dados, e NOAA pelo modelo HYSPLIT utilizados nas análises e ao Projeto ATMANTAR, edital $\mathrm{MCTI} / \mathrm{CNPq}$ do Ano Polar Internacional, processo $n^{\circ}$. 52.0182/2006-5 .

\section{Bibliografia}

ALBERS, J. R.; NATHAN, T. R. Pathways for Communicating the Effects of Stratospheric Ozone to the Polar Vortex: Role of Zonally AsymmetricOzone. J. Atmos. Sci., v. 69 (3), p. 785-801, 2012.

BENCHERIF, H.; EL AMRAOUI, L.; KIRGIS, G.; DE BELLEVUE, J. L.; HAUCHECORNE, A.; MZÉ, N.; PORTAFAIX, T.; PAZMINO, A.; GOUTAIL, F. Analysis of a rapid increase of stratospheric ozone during late austral summer 2008 over Kerguelen $\left(49.4^{\circ} \mathrm{S}, 70.3^{\circ} \mathrm{E}\right)$. Atmos. Chem. Phys., v. 11, p. 363-373, 2011.

BRINKSMA, E. J.; MEIJER, Y. J.; CONNOR, B. J.; MANNEY, G. L.; BERGWERFF, J. B.; BODEKER, G. E.; BOYD, I. S.; LILEY, J. B.; HOGERVORST, W.; HOVENIER, J. W.; LIVESEY, N. J.; SWART, D. P. J. Analysis of record-low ozone values during the 1997 winter over Lauder, New Zealand. Geophys. Res. Lett., v. 25, n. 15, p. 2785-2788, 1998.

CASICCIA, C.; ZAMORANO, F.; HERNANDEZ, A. Erythemal irradiance at the Magellan's region and Antarctic ozone hole 1999-2005. Atmosfera, v. 21 n.1, p. 1-12, 2008.

CHUBACHI, S. Preliminary result of ozone observations at Syowa Station from February, 1982 to January, 1983. Mem. Natl. Inst. Polar Res. Jpn. Spec., v. 34, p. 13-20, 1984.

CLAIN, G.; BARAY, J. L.; DELMAS, R.; KECKHUT, P.; CAMMAS, J. P. A lagrangian approach to analyse the tropospheric ozone climatology in the tropics: Climatology of stratosphere-troposphere exchange at Reunion Island. Atmos. Environ., v. 44, n. 7, p. 968-975, 2010.

DANIELSEN, E. F. Stratospheric-tropospheric exchange based upon radioactivity, ozone and potential vorticity. J. Atmos. Sci., v. 25, p. 502518, 1968.

FARMAN, J. C.; GARDINER, B. G.; SHANKLIN, J. D. Large losses of total ozone in Antarctica reveal seasonal $\mathrm{ClOx} / \mathrm{NOx}$ interaction. Nature. 315: 207-210, 1985.

GETTELMAN, A.; HOOR, P.; PAN, L. L.; RANDEL, W. J.; HEGGLIN, M. I.; BIRNER, T. The Extratropical Upper Troposphere and Lower Stratosphere. Rev. Geophys., v. 49, n. RG3003, 2011.

GUARNIERI, R. A.; PADILHA, L. F.; GUARNIERI, F. L.; ECHER, E.; MAKITA, K.; PINHEIRO, D. K.; SCHUCH, A.M.P.; BOEIRA, L. S.; SCHUCH, N.J. A study of the anticorrelations between ozone and UV-B radiation using linear and exponential fits in southern Brazil. Adv. Space Res., v. 34, p. 764768, 2004.

GUPTA, S.; LAL, S.; VENKATARAMANI, S.; RAJESH, T. A.; ACHARYA, Y. B. Variability in the vertical distribution of ozone over a subtropical site in India during a winter month. J. Atmos. Sol-Terr. Phy., v. 69, p. 1502-1512, 2007.

HOLTON, J. R.; HAYNES, P. H.; MCINTYRE, M. E.; DOUGLASS, A. R.; ROOD, R. B.; PFISTER, L. Stratosphere-troposphere

Rev.Geophys., v. 3, n. 3, p. 403-439, 1995.

HOSKINS, B. J.; McINTYRE, M. E.; ROBERTSON, A. W. On the use and significance of isentropic potential vorticity maps. Q. J. Roy. Metror. Soc., v. 111, p. 877-946, 1985. 
JING, P.; CUNNOLD, D. M.; YANG, E. S.; WANG, H. J. Influence of isentropic transport on seasonal ozone variations in the lower stratosphere and subtropical upper troposphere. J. Geophys. Res-Atmos., v. 110, n. D10110, 2005.

KALNAY, E.; KANAMITSU, M.; KISTLER, R.; COLLINS, W.; DEAVEN, D.; GANDIN, L.; IREDELL, M.; SAHA, S.; WHITE, G.; WOOLLEN, J.; ZHU, Y.; CHELLIAH, M.; EBISUZAKI, W.; HIGGINS, W.; JANOWIAK, J.; MO, K. C.; ROPELEWSKI, C.; WANG, J.; LEETMAA, A.; REYNOLDS, R.; JENNE, R.; JOSEPH, D. The NCEP/NCAR 40-year reanalysis project. Bull. Amer. Met. Soc., v. 77, n. 3, p. 437 - 471, 1996.

KIRCHHOFF, V. W. J. H.; SCHUCH, N. J.; PINHEIRO, D. K.; HARRIS, J. M. Evidence for an ozone hole perturbation at $30^{\circ}$ south. Atmos. Environ., v. 33, n. 9, p. 1481-1488, 1996.

KIRCHHOFF, V. W. J. H.; SAHAI, Y.; CASICCIA, C. A. R.; ZAMORANO, F.; VALDERRAMA, V. Observations of the 1995 ozone hole over Punta Arenas, Chile. J. Geophys. Res-Atmos., v. 102, n. D13, p. 1610916120, 1997.

LARY, D.; CHIPPERFILD, M.; PYLE, J.; NORTON, W.; RIISHOJGAARD. L. Treedimensional tracer initialization and general diagnostics using equivalent PV latitudepotential-temperature coordinates, Q. J. Roy. Metror. Soc., v. 121, p. 187-210, 1995.

LONDON, J. Observed distribution of atmospheric ozone and its variations. In: Whitten, R. C.; Prasad, S. S. ed. Ozone in the free atmosphere. New York: Van Nostrand Reinhold. cap. 1, p. $11-80.1985$.

MANNEY, G. L.; ZUREK, R. W.; NEIL, A. O.; SWINBANK, R. On the motion of air through the stratospheric polar vortex. J. Atmos. Sci., v. 51, p. 2973-2994, 1994.

MARCHAND, M.; BEKKI, S.; PAZMINO, A.; LEFÈVRE, F.; GODIN-BEEKMANN, S.; HAUCHECORNE, A. Model simulations of the impact of the 2002 Antarctic ozone hole on midlatitudes. J. Atmos. Sci., v. 62, p. 871-884, 2005.

NASH, E. R.; NEWMAN, P. A.; ROSENfiELD, J. E.; SCHOEBERL, M. E. An objective determination of the polar vortex using Ertel's potential vorticity. J. Geophys. Res., v. 101, p. 9471-9478, 1996.

PAZMINO, A. F.; GODIN-BEEKMANN, S.; LUCCINI, E. A.; PIACENTINI, R. D.; QUEL, E. J.; HAUCHECORNE, A. Increased UV radiation due to polar ozone chemical depletion and vortex occurrences at Southern Sub-polar Latitudes in the period [1997-2005]. Atmos. Chem. Phys., v. 8, n. 17, p. 5339-5352, 2008.

PERES, L. V.; KALL, E.; CRESPO, N.M; FONTINELE, J. L.; ANABOR, V.; PINHEIRO, D. K.; SCHUCH, N.J.; LEME, N.M.P. Caracterização sinótica do evento de Efeito Secundário do Buraco de Ozônio Antártico sobre o Sul do Brasil do dia 14/10/2008. Ciência e Natura, Ed. Suplementar, Universidade Federal de Santa Maria, p. 323 - 326, 2011.

PERES, L. V.; Crespo, N., M.; Da Silva, O.; K., HUPFER, N.; Anabor, V.; PINHEIRO, D. K.; SCHUCH, N. J.; LEME, N., P. Sinoptic weather system associate with influence of the Antartic Ozone Hole over South of Brazil at October, 13th, 2010. Annual Active Report 2011 National Institute of Science and Technology Antarctic Environmental Research., v. 1, p. 3033, 2012.

PERES, L. V. Efeito Secundário do Buraco de Ozônio Antártico Sobre o Sul do Brasil. Dissertação (Mestrado em Meteorologia). Universidade Federal de Santa Maria, 2013.

PEREZ, A.; DE CARCER, I. A.; TOCHO, J.O.; CRINO, E.; SANDOVAL, H. F. R.; BERNI, M. E.; DA SILVA, L.; HENRIQUES, D.; CUSSO, F.; JAQUE, F. The extent of the ozone hole over South America during the spring of 1993, 1994, and 1995. J. Phys. D Appl. Phys., v. 31, n. 7, p. 812-819, 1998.

PEREZ, A.; JAQUE, F. On the Antarctic origin of low ozone events at the South American continent during the springs of 1993 and 1994. Atmos. Environ., v. 32, n. 21, p. 3665-3668, 1998.

PEREZ, A.; CRINO, E.; DE CARCER, I. A.; JAQUE, F. Low-ozone events and threedimensional transport at midlatitudes of South America during springs of 1996 and 
1997. J. Geophys. Res-Atmos., v. 105, n. D4, p. 4553-4561, 2000.

PINHEIRO, D. K.; LEME, N. P.; PERES, L. V.; KALL, E. Influence of the Antarctic ozone hole over South of Brazil in 2008 and 2009. Annual Active Report 2010 - National Institute of Science and Technology Antarctic Environmental Research, v. 1, p. 33-37, 2011.

PINHEIRO, D. K.; PERES, L. V.; Crespo, N. M.; SCHUCH, N. J.; LEME, N., P. Influence of the Antarctic ozone hole over South of Brazil in 2010 and 2011. Annual Active Report 2011 National Institute of Science and Technology Antarctic Environmental Research, v. 1, p. 3438, 2012.

PLOEGER, F.; KONOPKA, P.; MUELLER, R.; FUEGLISTALER, S.; SCHMIDT, T.; MANNERS, J. C.; GROOSS, J. U.; GUENTHER, G.; FORSTER, P. M.; RIESE, M. Horizontal transport affecting trace gas seasonality in the Tropical Tropopause Layer (TTL). J. Geophys. Res-Atmos., v. 117, n. D09303, 2012.

PRATHER, M.; JAFFE, H. Global impact of the Antarctic ozone hole: chemical propagation. J. Geophys. Res-Atmos., v. 95, p. 3413-3492, 1990.

SCHOEBERL, M. R. Reconstruction of the constituent distribution and trends in the Antarctic polar vortex from ER-2 flight observations, J. Geophys. Res., v.94, p.16.81516.845, 1989.

SEMANE, N.; BENCHERIF, H.; MOREL, B.; HAUCHECORNE, A.; DIAB, R. D. An unusual stratospheric ozone decrease in Southern Hemisphere subtropics linked to isentropic airmass transport as observed over Irene (25.5 $\mathrm{S}$, 28.1 ${ }^{\mathrm{o}}$ E) in mid-May 2002. Atmos. Chem. Phys., v. 6, p. 1927-1936, 2006.

SIVAKUMAR, V.; PORTAFAIX, T.; BENCHERIF, H.; GODIN-BEEKMANN, S.; BALDY, S. Stratospheric ozone climatology and variability over a southern subtropical site: Reunion Island $\left(21^{\circ} \mathrm{S} ; 55^{\circ} \mathrm{E}\right)$. Annales of Geophysics, v. 25, p. 2321-2334, 2007.

SOLOMON, S. Stratospheric ozone depletion: a review of concepts and history. Rev.Geophy., v. 37, n. 3, p. 275-316, 1999.
SPRENGER, M.; WERNLI, H.; BOURQUI, M. Stratosphere-Troposphere Exchange and Its Relation to Potential Vorticity Streamers and Cutoffs near the Extratropical Tropopause. J. Atmos. Sci., v. 64, n. 5, p. 1587-1602, 2007.

STOHL, A.; WERNLI, H.; BOURQUI, M.; FORSTER, C.; JAMES, P.; LINIGER, M.A.; SEIBERT, P.; SPRENGER, M. A new perspective of stratosphere-troposphere exchange. Bull. Amer. Meteor. Soc., v. 84, p. 1565-1573, 2003.

WAUGH, D. W. Subtropical stratospheric mixing linked to disturbances in the polar vortices. Nature, v. 365, p. 535-537, 1993.

WAUGH, D.; PLUMB, R.; ATKINSON, R. J.; SCHOEBERL, M. R.; LAIT, L. R.; NEWMAN, P. A.; LOEWENSTEIN, M.; TOOHET, D.; AVALLONE, L.; WEBSTER, C.; MAY, R. Transport out of the lower stratospheric vortex by Rossby wave breaking. J. Geophys. ResAtmos., v. 99, p. 1071-1088, 1994. 